\title{
ANALYSIS OF AERODYNAMIC CHARACTERISTICS OF A SUPERCRITICAL AIRFOIL FOR LOW SPEED AIRCRAFT
}

\author{
P.Sethunathan ${ }^{1}$, M.Niventhran ${ }^{2}$, V.Siva ${ }^{2}$, R.Sadhan Kumar ${ }^{2}$ \\ ${ }^{1}$ Asst.Professor, Department of Aeronautical Engineering, Paavaai Group of Institutions, Namakkal \\ ${ }^{2} U G$ Students, Department of Aeronautical Engineering, Paavaai Group of Institutions, Namakkal
}

\begin{abstract}
The main goal of the proposed paper is the analysis of aerodynamic characteristics of an various supercritical airfoils like 0406, 0412, 0706 and 1006 influence on the dramatic improvement in lift and reduction of drag in low speed aircraft. In a precedent work, a cusp like structure at the trailing edge of an unsymmetrical airfoil produces a very high improvement in climbing performance of an aircraft.

The computational analysis of aerodynamic characteristics of supercritical airfoil design and analysis by using ICEM-CFD. In this work certain supercritical airfoils set on an ICEM-CFD without changing benchmark setup, in order to compare their coefficient of lift $\left(C_{L}\right)$ and coefficient of drag $\left(C_{D}\right)$ at various angle of attack $(\alpha)$. The pressure distribution was measured to verify global and local effects of airfoil structure. The test conducted in subsonic speed at flow velocity of $25 \mathrm{~m} / \mathrm{s}$. The result shows that certain supercritical airfoil (0406) configuration reduced the drag and improve coefficient of lift $\left(C_{L}\right)$ by 15-20\% compared with the baseline model.
\end{abstract}

Keywords: Supercritical airfoil, Lift Curve Slope, Coefficient of Lift, ICEM-CFD, Drag Reduction.

\section{INTRODUCTION}

\subsection{Flow Past a Supercritical Airfoil}

The flow over a finite wing is different from that of flow over an airfoil. Transonic Jet aircrafts fly at speed of 0.8-0.9 Mach number. At these speeds speed of air reaches speed of sound somewhere over the wing and compressibility effects start to show up. The free stream Mach number at which local sonic velocities develop is called critical Mach number. It is always better to increase the critical Mach number so that formation of shockwaves can be delayed. This can be done either by sweeping the wings but high sweep is not recommended in passenger aircrafts as there is loss in lift in subsonic speed and difficulties during constructions. So engineers thought of developing an airfoil which can perform this task without loss in lift and increase in drag. They increased the thickness of the leading edge and made the upper surface flat so that there is no formation of strong shockwave and curved trailing edge lower surface which increases the pressure at lower surface and accounts for lift.

\subsection{Scope and Objective of the Present Work}

In the present work, first of all we have analyzed the effect of number of airfoils to reduce the drag. We computed flow over the wing with different configurations of supercritical airfoils.. The four configurations of models are airfoil (0406, 0412, 0706, and 1006) results have been compared to the experimentally reported results from the literatures 0406 is good performance for $C_{L}, C_{D}$, and $C_{p}$ analyzed by ICEMCFD.

\section{LITERATURE REVIEW}

A concerted effort within the National Aeronautics and Space Administration (NASA) during the 1960's and 1970's was directed toward developing practical airfoils with twodimensional transonic turbulent flow and improved drag divergence Mach numbers while retaining acceptable lowspeed maximum lift and stall characteristics and focused on a concept referred to as the supercritical airfoil. This distinctive airfoil shape, based on the concept of local supersonic flow with isentropic recompression, was characterized by a large leading-edge radius, reduced curvature over the middle region of the upper surface, and substantial aft camber.

\subsection{Effects of Trailing-Edge Thickness}

The design philosophy of the supercritical airfoil required that the trailing-edge slopes of the upper and lower surfaces be equal. This requirement served to retard flow separation by reducing the pressure recovery gradient on the upper surface so that the pressure coefficients recovered to only slightly positive values at the trailing edge. For an airfoil with a sharp trailing edge, as was the case for early supercritical airfoils, such restrictions resulted in the airfoil being structurally thin over the aft region. Because of structural problems associated with sharp trailing edges and the potential aerodynamic advantages of thickened trailing edges for transonic airfoils, an exploratory investigation was made during the early development phases of the supercritical airfoil to determine the effects on the aerodynamic characteristics of thickening the trailing edge shows that increasing the trailing-edge thickness of an interim ill-percent-thick supercritical airfoil from 0 to 1.0 
percent of the chord resulted in a significant decrease in wave drag at transonic Mach numbers; however, this decrease was achieved at the expense of higher drag at subcritical Mach numbers.

\section{COMPUTATIONAL MODELLING OF FLUID FLOW}

CFD is one of the branches of fluid mechanics that uses numerical methods and algorithms to solve and analyze problems that involve fluid flows. Computers are used to perform the millions of calculations required to simulate the interaction of fluids and gases with the complex surfaces used in engineering. More accurate codes that can accurately and quickly simulate even complex scenarios such as supersonic or turbulent flows

4.

SUPERCRITICAL

AIRFOIL

\section{NOMENCLATURE}

$\begin{array}{lll}\text { Wing configuration } & \text { - SC (2) - } 0406 \\ \text { Wing configuration } & -\mathrm{SC}(2)-0412 \\ \text { Wing configuration } & -\mathrm{SC}(2)-0706 \\ \text { Wing configuration } & -\mathrm{SC}(2)-1006 \\ \text { Wing length } & - & 0.25 \mathrm{~m} \\ \text { Viscosity } & -1.8310 \mathrm{E}-05 \\ \text { Density } & -1.125 \mathrm{~kg} / \mathrm{m}^{3}\end{array}$

\section{NUMERICAL RESULTS AND GRAPHS}

In order to ensure that the numerical simulation of steady flow over airfoil at different orientations using ICEM-CFD is properly carried out; a few benchmark test cases for validation were simulated first.

\subsection{Inviscid Flow over a Supercritical Airfoil}

For the numerical simulation of flow over wing using commercial package ICEM-CFD has been used to solve the basic governing equations for velocities and other quantities.

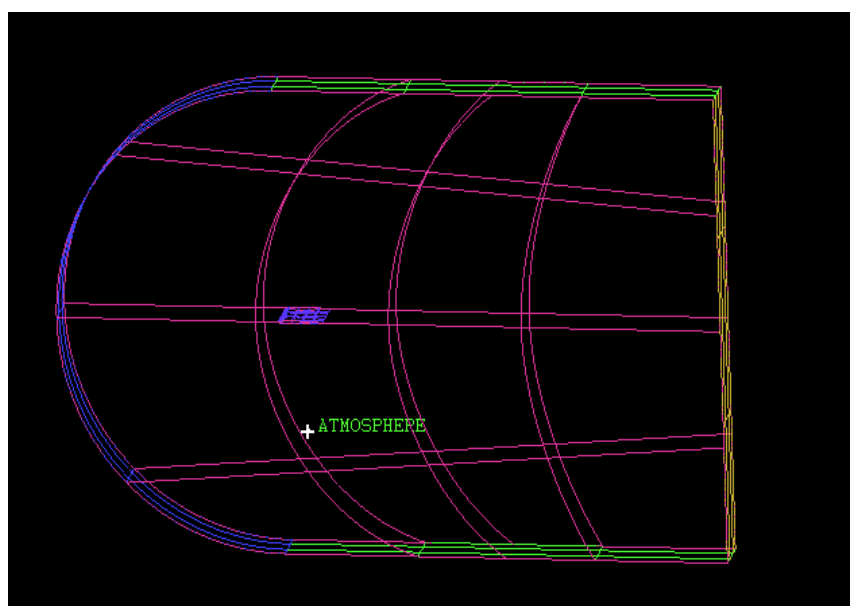

Fig.1. Domain wall

The schematic representation of the computational domain Structured grid in Cartesian coordinates is chosen, where $\mathrm{x}$ axis is along the free stream direction, $y$-axis is in the vertical direction, z-axis is in the span wise direction. The solution is started and allowed to converging and attains periodic nature.

\section{Conditions for the Inviscid Model}

\begin{tabular}{|c|c|c|}
\hline$*$ & INLET CONDITION & - INLET \\
\hline & OUTLET CONDITON & - OUTLET \\
\hline & AIRFOIL SURFACE & - WALL \\
\hline & \multicolumn{2}{|c|}{ TOP AND BOTTOM SURFACE - WALL } \\
\hline & SOLVER & - CFX \\
\hline & MODEL & - INVISCID \\
\hline & TIME & - STEADY FLOW \\
\hline & DENSITY & $-1.225 \mathrm{Kg} / \mathrm{m}^{3}$ \\
\hline
\end{tabular}

\subsection{Inviscid Flow over a Supercritical Airfoil (0406)}

\section{Meshing}

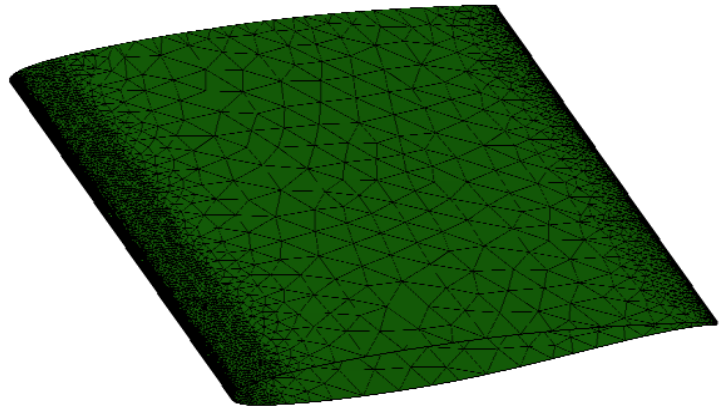

Fig. 2 Meshing of supercritical airfoil 0406

The flow over a wing for Reynolds number 2,50,000 is computed using ICEM-CFD simulating in supercritical airfoil. The variation of lift coefficient Vs angle of attack shown.Fig,7 As well as the variation of drag coefficient has shown Vs angle of attack shown in Fig.8 and $C_{L}$ Vs $C_{D}$ in Fig. 9 .

\subsection{Comparison of Inviscid Flow Supercritical}

\section{Airfoil (0406, 0412, 0706, 1006) Meshing}

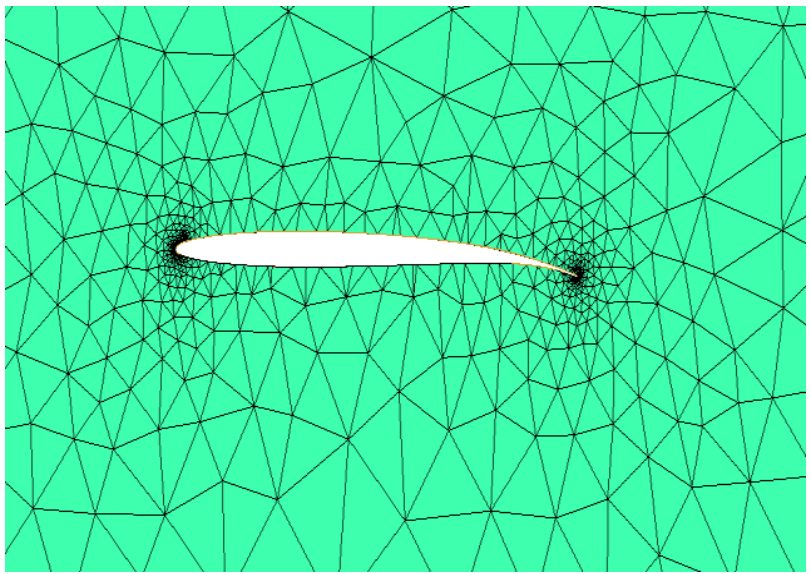

Fig. 3 Meshing Of Supercritical Airfoil 0406 


\subsection{Analyzing Flow Velocity}

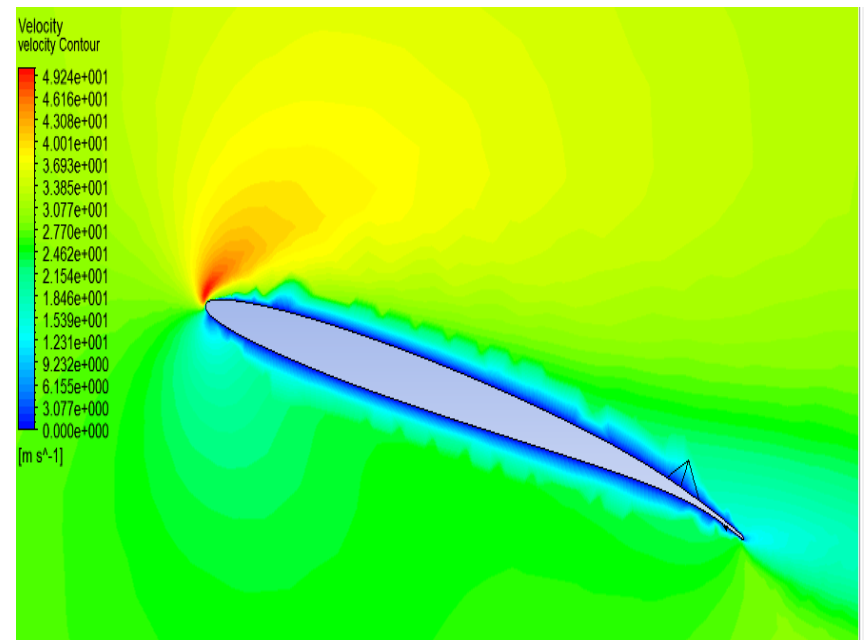

Fig. 4.Velocity distribution over a Supercritical Airfoil 0406

\subsection{Pressure}

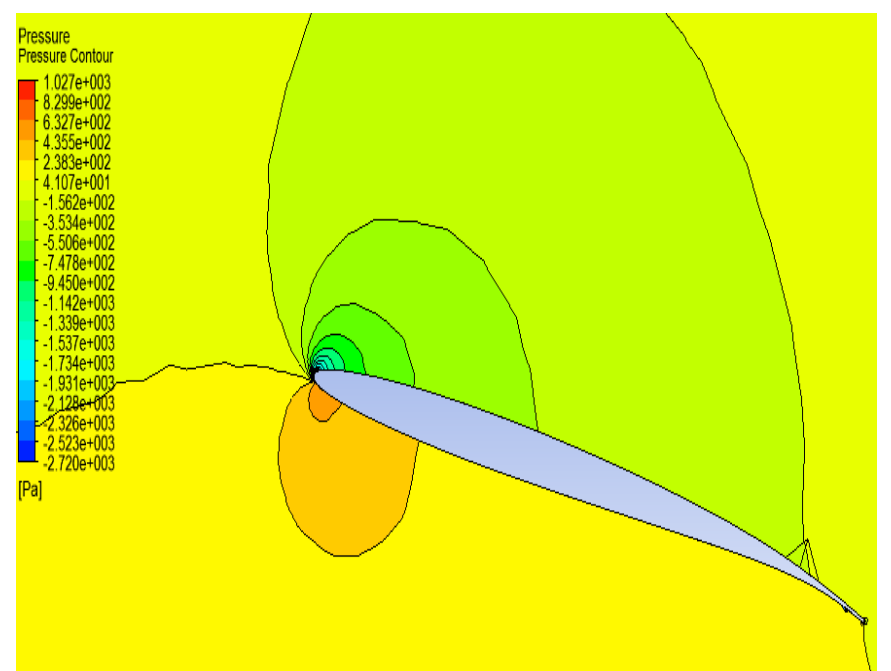

Fig. 5.Pressure distribution over a supercritical airfoil 0406

\subsection{Streamline Velocity}

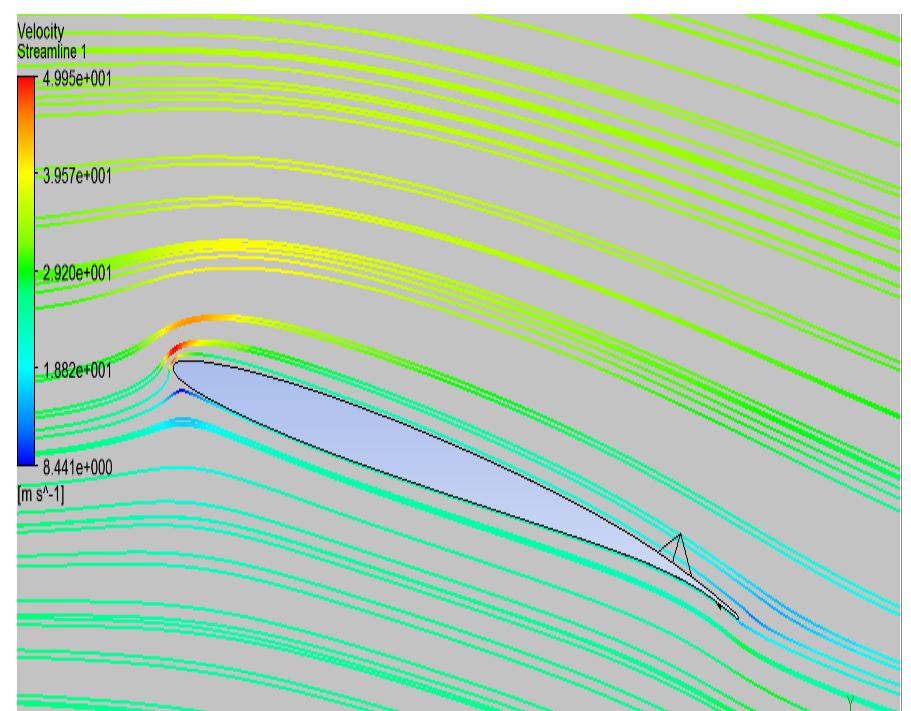

Fig. 6 Stream line velocity of supercritical airfoil 0406
Table 1 SC (2) - 0406

\begin{tabular}{|l|l|l|}
\hline $\mathbf{A O A}(\boldsymbol{\alpha})$ & $\mathbf{C}_{\mathbf{L}}$ & $\mathbf{C}_{\mathbf{D}}$ \\
\hline-5 & -0.426 & 0.0084 \\
\hline-3 & -0.197 & 0.0074 \\
\hline-1 & 0.033 & 0.0072 \\
\hline 0 & 0.148 & 0.0069 \\
\hline 1 & 0.263 & 0.0072 \\
\hline 3 & 0.472 & 0.0078 \\
\hline 5 & 0.561 & 0.0091 \\
\hline 7 & 0.634 & 0.0107 \\
\hline 9 & 0.692 & 0.0138 \\
\hline 11 & 0.733 & 0.0175 \\
\hline 13 & 0.758 & 0.0222 \\
\hline 15 & 0.766 & 0.0278 \\
\hline 16 & 0.763 & 0.0312 \\
\hline
\end{tabular}

Table 2 SC (2) - 0412

\begin{tabular}{|l|l|l|}
\hline $\mathbf{A O A}(\boldsymbol{\alpha})$ & $\mathbf{C}_{\mathbf{L}}$ & $\mathbf{C}_{\mathbf{D}}$ \\
\hline $\mathbf{- 5}$ & -0.304 & 0.0083 \\
\hline $\mathbf{- 3}$ & -0.065 & 0.0088 \\
\hline $\mathbf{- 1}$ & 0.174 & 0.0085 \\
\hline $\mathbf{0}$ & 0.293 & 0.0084 \\
\hline $\mathbf{1}$ & 0.412 & 0.0085 \\
\hline $\mathbf{3}$ & 0.651 & 0.0091 \\
\hline $\mathbf{5}$ & 0.83 & 0.0105 \\
\hline $\mathbf{7}$ & 0.962 & 0.0123 \\
\hline $\mathbf{9}$ & 1.062 & 0.0147 \\
\hline $\mathbf{1 1}$ & 1.131 & 0.0177 \\
\hline $\mathbf{1 3}$ & 1.169 & 0.0215 \\
\hline $\mathbf{1 5}$ & 1.176 & 0.0258 \\
\hline $\mathbf{1 6}$ & 1.168 & 0.0295 \\
\hline
\end{tabular}

Table 3 SC (2) - 0706

\begin{tabular}{|l|l|l|}
\hline $\mathbf{A O A}(\boldsymbol{\alpha})$ & $\mathbf{C}_{\mathbf{L}}$ & $\mathbf{C}_{\mathbf{D}}$ \\
\hline $\mathbf{- 5}$ & -0.634 & 0.0088 \\
\hline $\mathbf{- 3}$ & -0.381 & 0.0082 \\
\hline $\mathbf{- 1}$ & -0.127 & 0.008 \\
\hline $\mathbf{0}$ & 0 & 0.0078 \\
\hline $\mathbf{1}$ & 0.127 & 0.008 \\
\hline $\mathbf{3}$ & 0.381 & 0.0082 \\
\hline $\mathbf{5}$ & 0.634 & 0.0087 \\
\hline $\mathbf{7}$ & 0.851 & 0.0103 \\
\hline $\mathbf{9}$ & 1.004 & 0.0126 \\
\hline $\mathbf{1 1}$ & 1.104 & 0.0157 \\
\hline $\mathbf{1 3}$ & 1.158 & 0.0247 \\
\hline $\mathbf{1 5}$ & 1.172 & 0.0411 \\
\hline $\mathbf{1 6}$ & 1.161 & 0.0535 \\
\hline
\end{tabular}

Table 4 SC (2) - 1006

\begin{tabular}{|l|l|l|}
\hline $\mathbf{A O A}(\boldsymbol{\alpha})$ & $\mathbf{C}_{\mathbf{L}}$ & $\mathbf{C}_{\mathbf{D}}$ \\
\hline $\mathbf{- 5}$ & 0.073 & 0.0075 \\
\hline $\mathbf{- 3}$ & 0.08 & 0.0075 \\
\hline $\mathbf{- 1}$ & 0.087 & 0.009 \\
\hline $\mathbf{0}$ & 0.089 & 0.0093 \\
\hline $\mathbf{1}$ & 0.092 & 0.0099 \\
\hline $\mathbf{3}$ & 0.097 & 0.0117 \\
\hline
\end{tabular}




\begin{tabular}{|l|l|l|}
\hline $\mathbf{5}$ & 0.102 & 0.0144 \\
\hline $\mathbf{7}$ & 0.109 & 0.0254 \\
\hline $\mathbf{9}$ & 0.117 & 0.0432 \\
\hline $\mathbf{1 1}$ & 0.126 & 0.068 \\
\hline $\mathbf{1 3}$ & 0.137 & 0.1006 \\
\hline
\end{tabular}

\begin{tabular}{|l|l|l|}
\hline $\mathbf{1 5}$ & 0.148 & 0.1407 \\
\hline $\mathbf{1 6}$ & 0.154 & 0.1643 \\
\hline
\end{tabular}

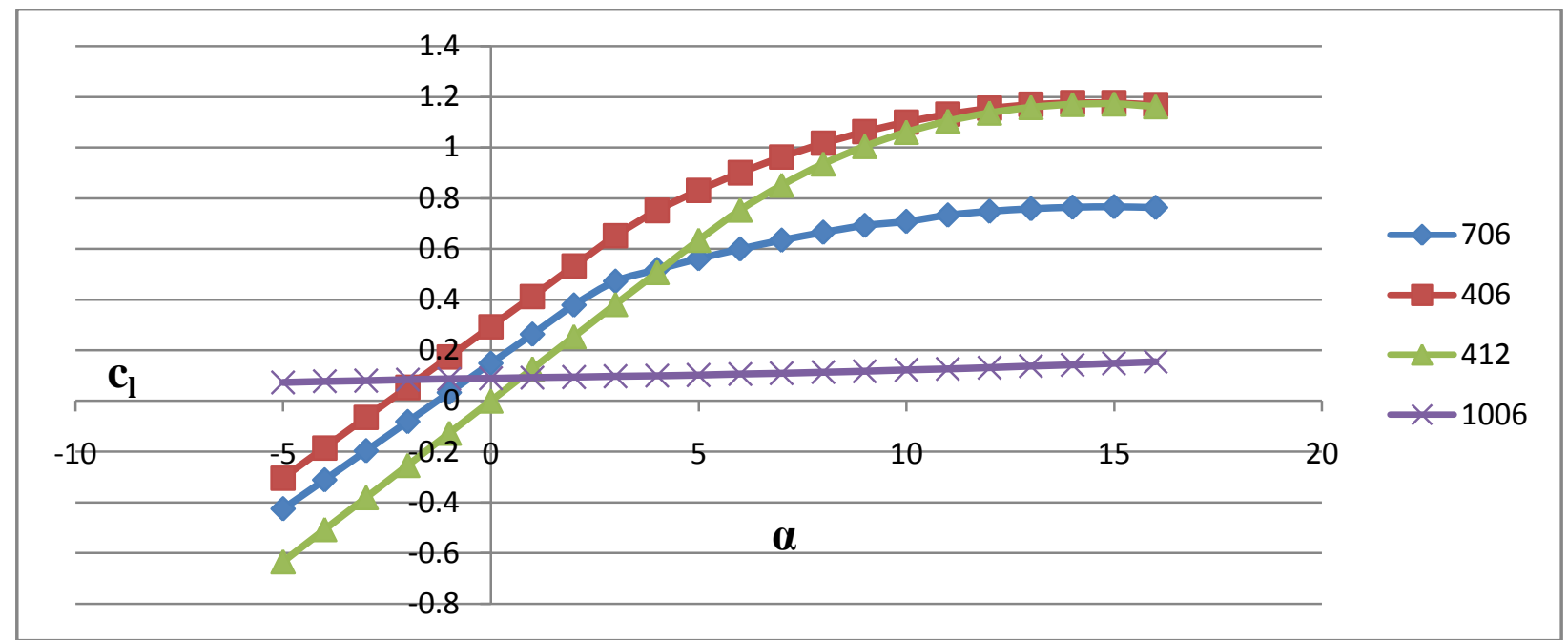

Fig. 7 Coefficient of lift $\left(C_{L}\right) V_{S}$ Angle of Attack $(\alpha)$

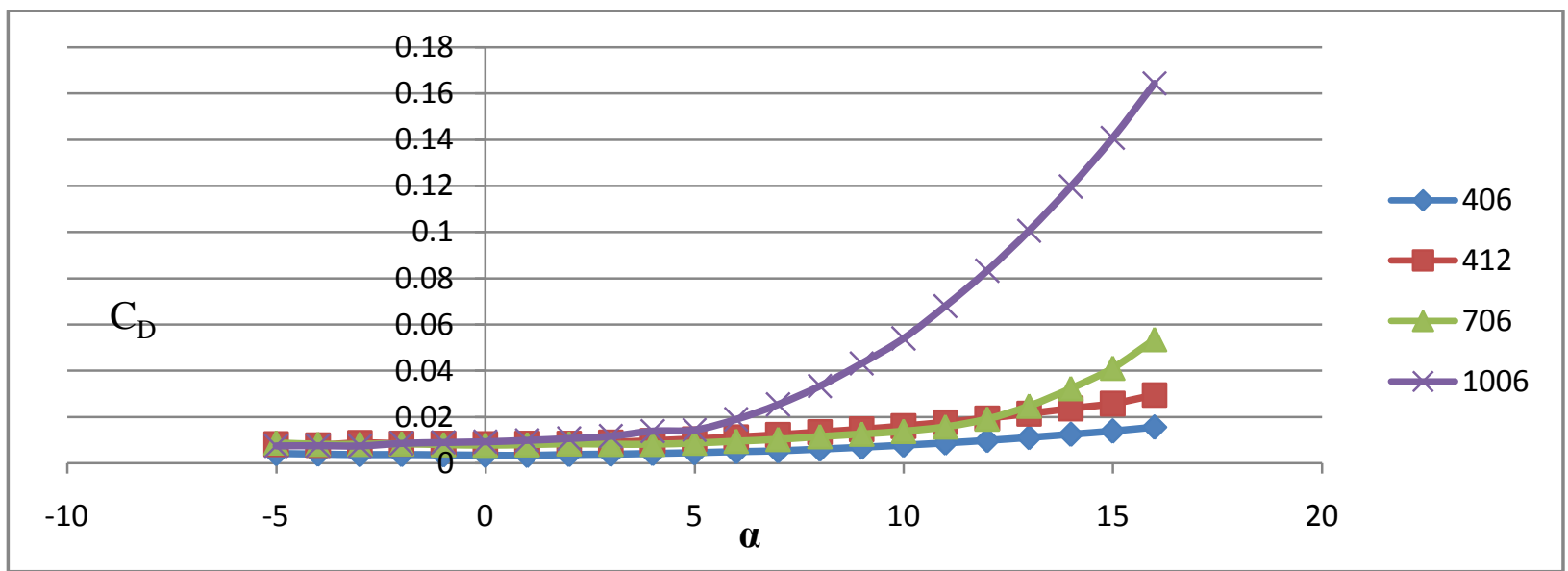

Fig. 8 Coefficient of drag $\left(C_{D}\right) V_{S}$ Angle of Attack $(\alpha)$

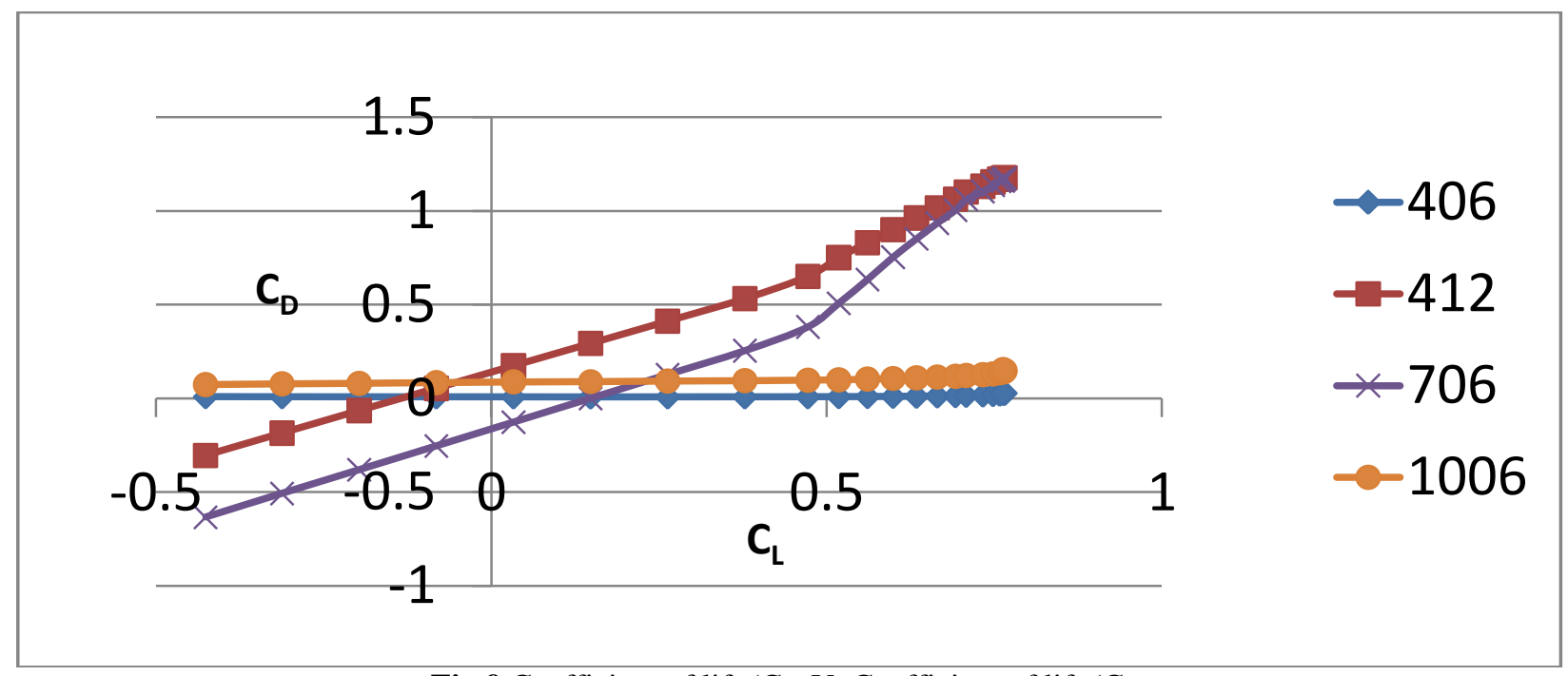

Fig 9 Coefficient of lift $\left(C_{L}\right) V_{s}$ Coefficient of lift $\left(C_{D}\right)$ 


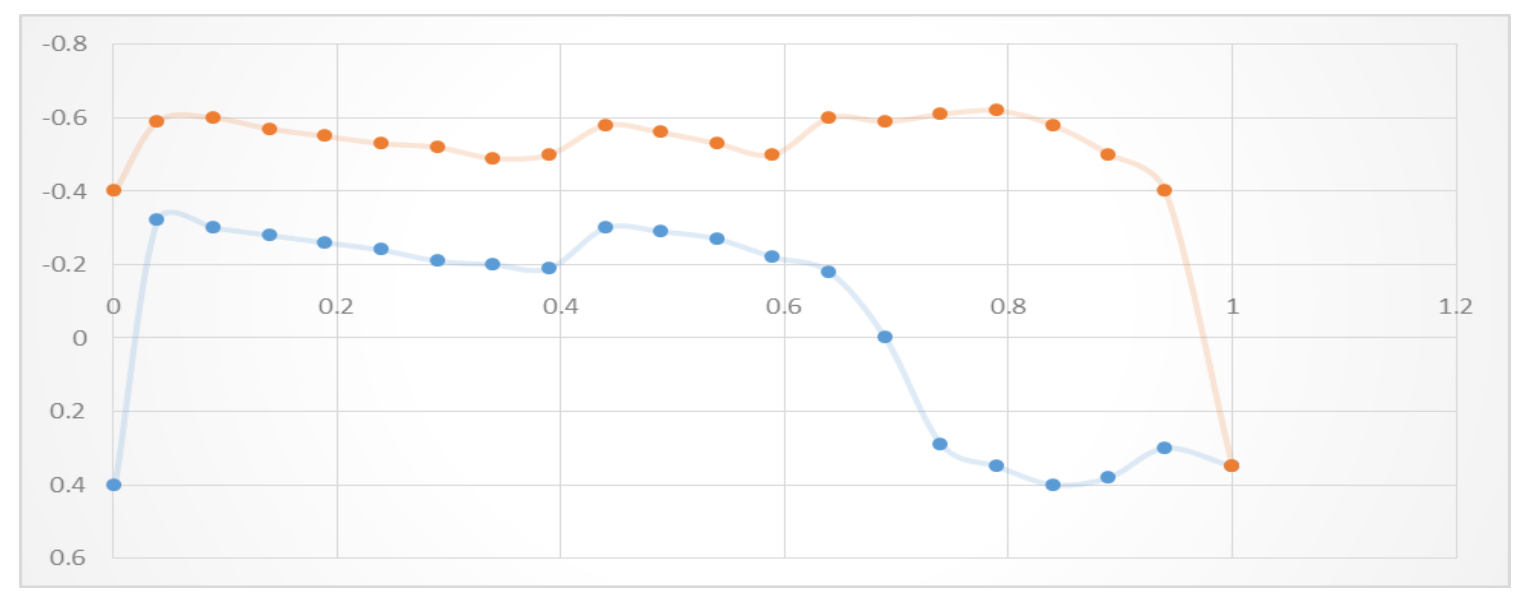

Fig.10. $C_{P} V_{S}$ LOCATION

\section{CONCLUSIONS}

Computational investigations have been performed to examine the effectiveness of the various supercritical airfoil mounted at varying angle to improve the performance of a wing in subsonic flow. Combining the Computational analysis measurement results with the experimental result, the following are presented for analysis performance due to various supercritical airfoils.

This distinctive airfoil shape, based on local supersonic flow with isentropic recompression, was characterized by a large leading-edge radius, reduced curvature over the middle region of the upper surface, and substantial aft camber. This report has summarized the NASA supercritical airfoil development program in a chronological fashion, discussed some of the airfoil design guidelines, and presented coordinates of a matrix of family-related supercritical airfoils with thicknesses of 2 to 18 percent and design lift coefficients from 0 to 1.0 .

From supercritical airfoil 0406 is we can conclude that produces more lift and lesser drag compare to the other supercritical airfoil.

\section{REFERENCES}

[1] McGhee, Robert J.; and Beasley, William D.: LowSpeed Aerodynamic Characteristics of a 17-PercentThick Airfoil Section Designed for General Aviation Applications. NASA TN D-7428, 1973.

[2] Whitcomb, Richard T.: Review of NASA Supercritical Airfoils. ICAS Paper No. 74-10, Aug. 1974

[3] McGhee, Robert J.; and Beasley, William D.: Effects of Thickness on the Aerodynamic Characteristics of an Initial Low-Speed Family of Airfoils for General Aviation Applications. NASA TM X-72843,1976

[4] McGhee, Robert J.; and Beasley, William D.: LowSpeed Wind-Tunnel Results for a Modified 13Percent- Thick Airfoil. NASA TM X-74018, 1977

[5] McGhee, Robert J.; and Beasley, William D.: WindTunnel Results for an Improved 21-Percent-Thick
Low- Speed Airfoil Section. NASA TM-78650, 1978.

[6] McGhee, Robert J.; and Beasley, William D." LowSpeed Aerodynamic Characteristics of a 13-PercentThick Medium-Speed Airfoil Designed for General Aviation Applications. NASA TP-1498, 1979.

[7] McGhee, Robert J.; and Beasley, William D.: LowSpeed Aerodynamic Characteristics of a 17-PercentThick Medium-Speed Airfoil Designed for General Aviation Applications. NASA TP-1786, 1980.

[8] McGhee, Robert J.; and Beasley, William D.: WindTunnel Results for a Modified 17-Percent-Thick Low- Speed Airfoil Section. NASA TP-1919, 1981.

[9] Ferris, James D.; McGhee, Robert J.; and Barnwell, Richard W.: Low-Speed Wind-Tunnel Results for Symmetrical NASA LS (1)-0013 Airfoil. NASA TM4003, 1987.14

[10] . Whitcomb, Richard T.; and Clark, Larry R.: An Airfoil Shape for Efficient Flight at Supercritical Mach Numbers. NASA TM X-1109, 1965. 